\title{
Hilly neighborhoods are associated with increased risk of weight gain among older adults in rural Japan: a 3-years follow-up study
}

\author{
Kenta Okuyama ${ }^{1 *} \mathbb{D}$, Takafumi Abe ${ }^{1}$, Tsuyoshi Hamano ${ }^{2}$, Miwako Takeda ${ }^{1}$, Kristina Sundquist ${ }^{3}$, Jan Sundquist ${ }^{3}$
} and Toru Nabika ${ }^{4}$

\begin{abstract}
Background: Neighborhood environments have been regularly associated with the weight status. Although the evidence is mostly limited to adults residing in western urban settings, the weight status of older adults living in rural areas is also assumed to be significantly affected by their neighborhood environments. This study aimed to identify environmental attributes specific to rural areas that could affect the risk of longitudinal weight gain among older adults ( $\geq 65$ years) in Japan.

Methods: We examined five environmental attributes, i.e., land slope, public transportation accessibility, residential density, intersection density, and the availability of parks and recreational centers, measured by the geographic information system. Our analysis was based on 714 subjects participated in Shimane Community-based Healthcare Research and Education study in 2012 and 2015. Multinomial logistic regression model was conducted to examine the association between each neighborhood environmental attribute and weight change status (gain, loss and unchanged).
\end{abstract}

Results: We observed a significant increase in the risk of weight gain as the steepness of the neighborhood land slope increased. There was no significant association between other environmental attributes and risk of weight gain as well as weight loss among older adults.

Conclusion: Living in hilly neighborhoods was associated with increased risk of weight gain among rural Japanese older adults. Future research should consider region-specific environmental attributes when investigating their effect on older adults' weight status.

Keywords: Neighborhood, Rural, Slope, Weight change, Older adults

\section{Background}

Weight change among older adults increases their mortality risk [1]. This has been established in several longitudinal studies [2,3]. The burdens of excess weight were estimated for causing 3.4 million deaths globally [4], and significant increase of medical cost burden attributed to excess weight has been observed in the US and east Asian countries, including Japan with the consistent rise

\footnotetext{
*Correspondence: ko215ok@gmail.com

${ }^{1}$ Center for Community-based Healthcare Research and Education (CoHRE), Organization for Research and Academic Information, Shimane University, 223-8 Enya-cho, Izumo-shi, Shimane 693-8501, Japan Full list of author information is available at the end of the article
}

of obesity prevalence [5-7]. Neighborhood environments are known to have substantial impacts on the health and functioning of older adults, and also weight change [8]. While several studies [9-11] have longitudinally assessed the change in weight-related measures and neighborhood environments, one of them [9] have found significant associations between the neighborhood environment and weight status among older adults. These studies were mostly conducted in western urban settings, and the evaluation of neighborhood environments is primarily based on urban environmental attributes such as residential density, land use mix, street connectivity, and retail floor ratio, measured by geographic information system 
(GIS) [12]. These urban environmental measures are not thought to be associated with the health behaviors of the rural population $[13,14]$ and are rarely applicable in the assessment of rural neighborhood environments [15].

Rural residents are at a higher risk of chronic health conditions such as cardiovascular disease and some forms of cancer, as well as modifiable risk factors such as physical inactivity and obesity when compared to those of urban and suburban counterparts [16, 17]. For example, lower residential density and population density have been consistently found to be associated with physical inactivity internationally [18-20]. Furthermore, a higher percentage of aging population is more often observed in rural settings across the world [21]. Despite these, there is a lack of studies in rural settings, especially among elderly people.

Japan is defined as a "super-aged society," a term used to describe a society where more than $21 \%$ of the total population is aged above 65 years according to the standard definition by the United Nations and World Health Organization. As of 2015, the aging rate reached an unprecedented level of $26 \%$ in Japan and was the highest in the world [22]. Although there are a few studies in Japan which have examined neighborhood environmental characteristics, weight status, and other healthrelated outcomes among older adults using GIS [23-27], those are limited in urban settings. In those studies, some environmental attributes, such as access to public transit, elevation, and land slope were found to be associated with physical activity, hypertension, and diabetes [25-27]. There is a growing interest to measure not only built environment but also natural environment as those are thought to have significant impact on population health, especially in rural settings where urban attributes are not applicable [28]. Slope land is a unique geographic feature of Japan and the cause for hilly neighborhoods [29]. Identifying environmental attributes in rural settings is critical to enact policy and environmental level intervention in order to improve the health of vulnerable population. From these backgrounds, the purpose of this study is to identify environmental attributes specific to vulnerable areas, i.e., rural areas, that can affect weight change among Japanese older adults by longitudinal study design.

\section{Methods}

\section{Data}

This study used longitudinal data of older adults ( $\geq 65$ years) who participated in our Shimane CoHRE study. Shimane CoHRE is a cohort study in collaboration with three municipalities in the Shimane Prefecture to identify potential factors of healthy aging among rural residents by investigating their lifestyle habits and any chronic conditions they may have had. Shimane prefecture is a rural mountainous region located in western Japan. In Japan, urban area refers to the area with basic unit census block with population density 4000 or more per square $\mathrm{km}$, and whose total population exceeds 5000 . Rural-mountainous areas defined as the areas whose $50-80 \%$ or more of the land is covered by the forest, and cultivated lands are below $10 \%$ of total land areas and are mostly hilly [30]. Shimane has the third highest aging rate among the 47 prefectures in Japan (Total population in Shimane: 694 352; Japan: 12483 901, the percentage of the population aged 65 years and above in Shimane: $32.5 \%$; Japan: $26.6 \%$, population density: 103.5 person $/ \mathrm{km}^{2}$; Japan: 340.8 person $/ \mathrm{km}^{2}$ ) [31]. The current study utilized the CoHRE data for 2012 (baseline) and 2015 (follow-up). The study subjects were those who participated in the annual health checkup, and agreed to participate in Shimane CoHRE study. Among 2147 people, 1630 subjects ( $\geq 65$ years) participated in the baseline study. Among them, 926 subjects participated in the follow-up survey in 2015, of which, 212 subjects were excluded since data for relevant variables were missing. In the final study, there were 714 subjects. We examined whether there are differences in terms of basic characteristics, i.e., gender, age, and baseline weight, between the subjects included and not included in the study. There was no significant difference in terms of gender and baseline weight ( $p=0.081, p=0.158$, respectively). Although there was statistically significant difference in terms of age $(p<0.001)$, the difference in median age value was only 1.0 (those included: 70.0, those not included: 71.0). All subjects have not moved their residential location over 3 years. The study protocol and procedure used to secure the informed consent of the participants were approved by the Ethics Committee of Shimane University \#2888.

\section{Outcome variable}

The weight of each participant was measured objectively by trained healthcare practitioners. These data were used as primary outcomes. The weight change $\geq 2.5 \mathrm{~kg}$ over 3 years was used as the cutoff point for weight gain and loss statuses in accordance with previous studies $[2,3$, 32].

\section{Exposure variable}

We used a total of five environmental attributes, i.e., land slope, public transportation accessibility, residential density, intersection density, and park and recreation center availability, within $400 \mathrm{~m}$ and $800 \mathrm{~m}$ network buffers from each residential point of the subjects in the study. We set multiple buffers of 400 and $800 \mathrm{~m}$ based on actual street network data, which are commonly defined 
as appropriate activity spaces for people in neighborhood studies [12]. The mean land slope, which expresses the steepness of hilly neighborhoods [23, 27], was computed based on the elevation and degree of Slope 5th Mesh Data (as of 2011). We computed the mean land slope within network buffers by calculating the value of slope stored in each $50 \mathrm{~m}$ grid which intersected with network buffers. Hilliness was represented by an angular unit: degree. These data were obtained from the National Land Numerical Information (NLNI), which is publicly available GIS data administered by the National Land Information Division, National Spatial Planning, and Regional Policy Bureau of Japan. Further, accessibility of public transportation, was computed using the number of bus stops and railway stations within each network buffer based on the bus stop and railway station point data as of 2010, available from NLNI. The residential density was computed using the number of households within each network buffer based on the data of the basic block unit households (as of 2010), which is the smallest Japanese census statistics area from the ArcGIS data collection by Esri Corporation, Tokyo, Japan (Esri Japan). The value of number of households stored in point data were summed which fallen within network buffers, and was used as residential density. Intersection density was computed as street connectivity using the number of intersections with three or more legs within each network buffer, based on the road network data collected by Esri Japan for 2012. The availability of park and recreational centers was computed within each network buffer based on the data on city parks as of 2011 and cultural facilities as of 2012, both obtained as point data from NLNI. All spatial analyses were conducted using ArcGIS 10.0 (Esri Inc., Redland, CA).

\section{Covariates}

In the analysis, age (continuous), gender (male vs. female), baseline body weight (continuous), smoking habits (smoker vs. nonsmoker), drinking habits (drinker vs. non-drinker), having a driving license (yes vs. no), and educational attainment were included as covariates. All of this information was collected using self-reported questionnaires and face-to-face interviews. Data on educational attainment were collected through face-to-face interviews by talking to the participants about the duration of their education, and 12 years (high school graduate and above) was used as the cutoff.

\section{Statistical analysis}

Descriptive statistics showed the characteristics of all study subjects. Summary statistics and correlation analysis were conducted for five neighborhood environment variables. Multinomial logistic regression was conducted to predict that the odds of weight gain or weight loss, when compared to weight change status, would remain unchanged over 3 years. The exposure variables, namely, the neighborhood environment variables, were all included separately in the model with both continuous and categorical measures. The mean land slope, residential density, and intersection density were included in the analysis as a quartile categorical value. The public transit stop density and park and recreational center were included in the analysis as a binary categorical value (more than one versus none) since these measures were absent in the neighborhoods of many subjects. To avoid multicollinearity among exposure variables, each variable was included separately in the model by adjusting for all covariates. All statistical analyses were conducted using R 3.4.3.

\section{Results}

Basic characteristics of study subjects are shown in Table 1. The weights of $72.0 \%$ of the people did not change by more than $2.5 \mathrm{~kg}$ over 3 years, while $9.2 \%$ of the people gained more than $2.5 \mathrm{~kg}$, and $18.8 \%$ of people lost more than $2.5 \mathrm{~kg}$. The median age was similar

Table 1 Characteristics of the participants by weight change status

\begin{tabular}{lccr}
\hline & \multicolumn{2}{l}{ Weight change status $(\mathbf{2} \mathbf{2 . 5} \mathbf{~ k g})$ for $\mathbf{3}$ years } & Lost \\
\cline { 2 - 4 } & \multicolumn{1}{l}{ Unchanged } & \multicolumn{1}{l}{ Gained } & $134(18.8)$ \\
$\mathrm{n}(\%)$ & $514(72.0)$ & $66(9.2)$ & $71.0(68.0,71.0)$ \\
Age [median (IQR)] & $70.0(68.0,71.0)$ & $70.0(67.0,73.0)$ & $45 / 89(33.6 / 66.4)$ \\
Gender: male/female (\%) & $191 / 323(37.2 / 62.8)$ & $33 / 33(50.0 / 50.0)$ & $53.75(49.8,59.6)$ \\
Weight [median (IQR)] & $52.25(46.4,57.9)$ & $54.80(48.1,60.2)$ & $129 / 5(96.3 / 3.7)$ \\
Smoking: non-smoker/smoker (\%) & $485 / 29(94.4 / 5.6)$ & $50 / 16(75.8 / 24.2)$ & $57 / 77(42.5 / 57.5)$ \\
Drinking: non-drinker/drinker (\%) & $245 / 269(47.7 / 52.3)$ & $38 / 28(57.6 / 42.4)$ & $33 / 101(24.6 / 75.4)$ \\
Education: $\geq 12$ years/<12 years (\%) & $159 / 355(30.9 / 69.1)$ & $21 / 45(31.8 / 68.2)$ & $32 / 102(23.9 / 76.1)$ \\
Driving license: no/yes (\%) & $117 / 397(22.8 / 77.2)$ & $15 / 51(22.7 / 77.3)$ & 3
\end{tabular}


across people whose weight status remained unchanged [70.0 years, interquartile range $(\mathrm{IQR})=68.0,71.0]$, gained (70.0 years, $\mathrm{IQR}=67.0,73.0$ ), and lost (71.0 years, $\mathrm{IQR}=68.0,71.0)$. The median weight at the baseline was also similar across all groups. Around one-third of the participants had more than 12 years of education across all groups. More than $75 \%$ of participants had driving licenses across all groups.

Table 2 summarizes the five neighborhood environment variables and their correlations for $400 \mathrm{~m}$ and $800 \mathrm{~m}$ network buffers. The land slope was negatively associated with all other environment variables. Residential density and intersection density were highly correlated in both $400 \mathrm{~m}$ and $800 \mathrm{~m}$ network buffers $(\mathrm{r}=0.75$, and $\mathrm{r}=0.86$, respectively).

Table 3 presents the results of the multinomial logistic regression analysis for weight change status. The model included the neighborhood environment variables as categorical measures showed that land slope was significantly associated with weight gain for both $400 \mathrm{~m}$ and $800 \mathrm{~m}$ network buffers. While there was no significant increase in the odds of weight gain in the second quartile, taking the first quartile as a reference, there was a significant increase in the odds of weight gain in

Table 2 Summary of five neighborhood environment variables and their correlation coefficient

\begin{tabular}{|c|c|c|c|c|c|c|c|}
\hline \multirow[t]{2}{*}{$\mathbf{N}$} & 714 & 714 & 1 & 2 & 3 & 4 & 5 \\
\hline & Mean (SD) & \multicolumn{6}{|l|}{ Median (IQR) } \\
\hline \multicolumn{8}{|l|}{400 m network buffer } \\
\hline 1. Land slope & $9.62(4.66)$ & $8.87(5.82,12.64)$ & 1.00 & & & & \\
\hline 2. Public transit stop density & $1.16(1.57)$ & $1.00(0.00,2.00)$ & -0.33 & 1.00 & & & \\
\hline 3. Residential density & $111.77(134.59)$ & $64.00(26.00,142.00)$ & -0.58 & 0.64 & 1.00 & & \\
\hline 4. Intersection density & $5.37(5.60)$ & $4.00(2.00,7.00)$ & -0.57 & 0.63 & 0.75 & 1.00 & \\
\hline 5. Park and recreational center & $0.22(0.80)$ & $0.00(0.00,0.00)$ & -0.24 & 0.19 & 0.27 & 0.33 & 1.00 \\
\hline \multicolumn{8}{|l|}{800 m Network buffer } \\
\hline 1. Land slope & $10.77(4.75)$ & $10.08(6.63,14.52)$ & 1.00 & & & & \\
\hline 2. Public transit stop density & $2.37(2.59)$ & $2.00(1.00,3.00)$ & -0.23 & 1.00 & & & \\
\hline 3. Residential density & $209.11(232.70)$ & $126.00(47.25,274.00)$ & -0.60 & 0.62 & 1.00 & & \\
\hline 4. Intersection density & $12.13(11.76)$ & $9.00(4.00,16.75)$ & -0.63 & 0.64 & 0.86 & 1.00 & \\
\hline 5. Park and recreational center & $0.70(1.42)$ & $0.00(0.00,1.00)$ & -0.35 & 0.37 & 0.55 & 0.60 & 1.00 \\
\hline
\end{tabular}

Table 3 Multinomial logistic regression for the association between weight status and each neighborhood environmental attributes

\begin{tabular}{|c|c|c|c|c|c|c|c|c|}
\hline \multirow[t]{3}{*}{ Neighborhood environmental attributes } & \multicolumn{4}{|c|}{$400 \mathrm{~m}$ Network buffer } & \multicolumn{4}{|c|}{$800 \mathrm{~m}$ Network buffer } \\
\hline & \multicolumn{2}{|c|}{ Weight gain } & \multicolumn{2}{|c|}{ Weight loss } & \multicolumn{2}{|c|}{ Weight gain } & \multicolumn{2}{|c|}{ Weight loss } \\
\hline & OR & $95 \% \mathrm{Cl}$ & OR & $95 \% \mathrm{Cl}$ & OR & $95 \% \mathrm{Cl}$ & OR & $95 \% \mathrm{Cl}$ \\
\hline Land slope (2nd vs. 1st) & 1.295 & $(0.527,3.184)$ & 0.798 & $(0.460,1.385)$ & 3.013 & $(1.207,7.521)$ & 1.223 & $(0.712,2.101)$ \\
\hline Land slope (3rd vs. 1st) & 2.351 & $(1.048,5.275)$ & 1.007 & $(0.591,1.716)$ & 3.399 & $(1.404,8.229)$ & 1.069 & $(0.617,1.852)$ \\
\hline Land slope (4th vs. 1st) & 2.578 & $(1.175,5.654)$ & 0.837 & $(0.480,1.461)$ & 3.084 & $(1.295,7.343)$ & 0.968 & $(0.561,1.671)$ \\
\hline Public transit stop density ( $\geq 1$ vs. 0 ) & 0.607 & $(0.356,1.035)$ & 0.851 & $(0.574,1.261)$ & 0.847 & $(0.466,1.538)$ & 1.151 & $(0.716,1.848)$ \\
\hline Residential density (2nd vs. 1st) & 0.566 & $(0.270,1.189)$ & 0.830 & $(0.487,1.416)$ & 0.602 & $(0.292,1.239)$ & 1.389 & $(0.822,2.347)$ \\
\hline Residential density (3rd vs. 1st) & 0.414 & $(0.190,0.899)$ & 0.622 & $(0.363,1.065)$ & 0.417 & $(0.197,0.880)$ & 0.682 & $(0.387,1.202)$ \\
\hline Residential density (4th vs. 1st) & 0.952 & $(0.473,1.913)$ & 0.912 & $(0.513,1.618)$ & 0.803 & $(0.390,1.654)$ & 1.125 & $(0.630,2.009)$ \\
\hline Intersection density (2nd vs. 1st) & 0.491 & $(0.221,1.092)$ & 1.433 & $(0.854,2.406)$ & 0.834 & $(0.418,1.663)$ & 0.850 & $(0.496,1.454)$ \\
\hline Intersection density (3rd vs. 1st) & 0.725 & $(0.349,1.508)$ & 1.302 & $(0.754,2.248)$ & 0.568 & $(0.260,1.239)$ & 1.152 & $(0.686,1.936)$ \\
\hline Intersection density (4th vs. 1st) & 0.660 & $(0.323,1.349)$ & 1.112 & $(0.646,1.915)$ & 0.835 & $(0.408,1.711)$ & 0.851 & $(0.484,1.495)$ \\
\hline Park and recreational center ( $\geq 1$ vs. 0 ) & 0.529 & $(0.179,1.557)$ & 1.081 & $(0.569,2.052)$ & 1.432 & $(0.803,2.554)$ & 1.045 & $(0.671,1.630)$ \\
\hline
\end{tabular}

Each neighborhood environmental attribute was included separately

Age, gender, baseline weight, smoking, drinking, education attainment, and driving license were adjusted

Italic shows significance $p<0.05$ 
the third and fourth quartiles [Odds ratio (OR) 2.351, (95\% Confidence interval (CI) 1.048, 5.275); OR 2.578, (95\% CI 1.175, 5.654)] for the $400 \mathrm{~m}$ network buffer. The result indicates that living in the area with third or fourth quartile of slope had 2.4 or 2.6 times higher odds of weight gain. For the $800 \mathrm{~m}$ network buffer, there was a significant increase in the odds of weight gain in the second, third, and fourth quartiles when compared to the first quartile [OR 3.013, (95\% CI 1.207, 7.521); OR 3.399, (95\% CI 1.404, 8.229), OR 3.084, (95\% CI $1.295,7.343)]$. No significant association was observed between weight loss and land slope. There was a significant decrease of odds in the third quartile of residential density within the 400 and $800 \mathrm{~m}$ network buffer when compared to the first quartile [OR 0.414, (95\% CI 0.190, 0.899), OR 0.417, (95\% CI 0.197, 0.880), respectively]. Public transit stop density, intersection density, and the availability of parks and recreational centers were not significantly associated with either weight gain or loss for both the 400 and $800 \mathrm{~m}$ network buffers. The model, including neighborhood environment variables as the continuous measure, showed that the odds of weight gain were positively associated with land slope (results not shown). Figure 1 shows the predicted probabilities of weight change with neighborhood land slope within $400 \mathrm{~m}$ network buffer. It shows that probabilities of weight gain increases as degree of land slope increases. When the network buffers were extended to $800 \mathrm{~m}$, the significant association between weight gain and land slope was still observed, and similar trend was observed (Fig. 2). No significant association was observed between weight loss and land slope for both $400 \mathrm{~m}$ and $800 \mathrm{~m}$ network buffers. Other neighborhood environment variables were not associated with either

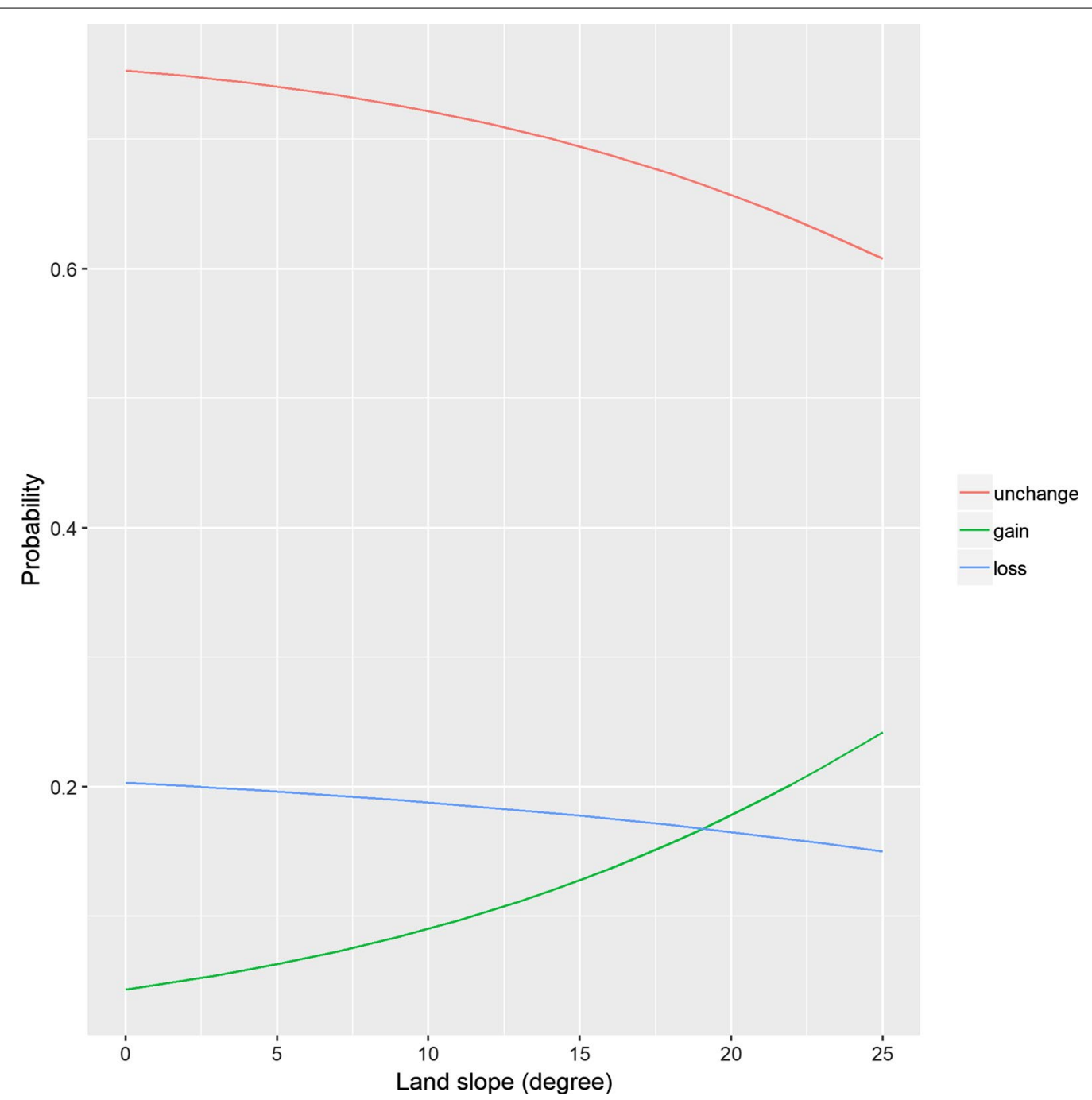

Fig. 1 Predicted probabilities of weight change by land slope in $400 \mathrm{~m}$ network buffer 


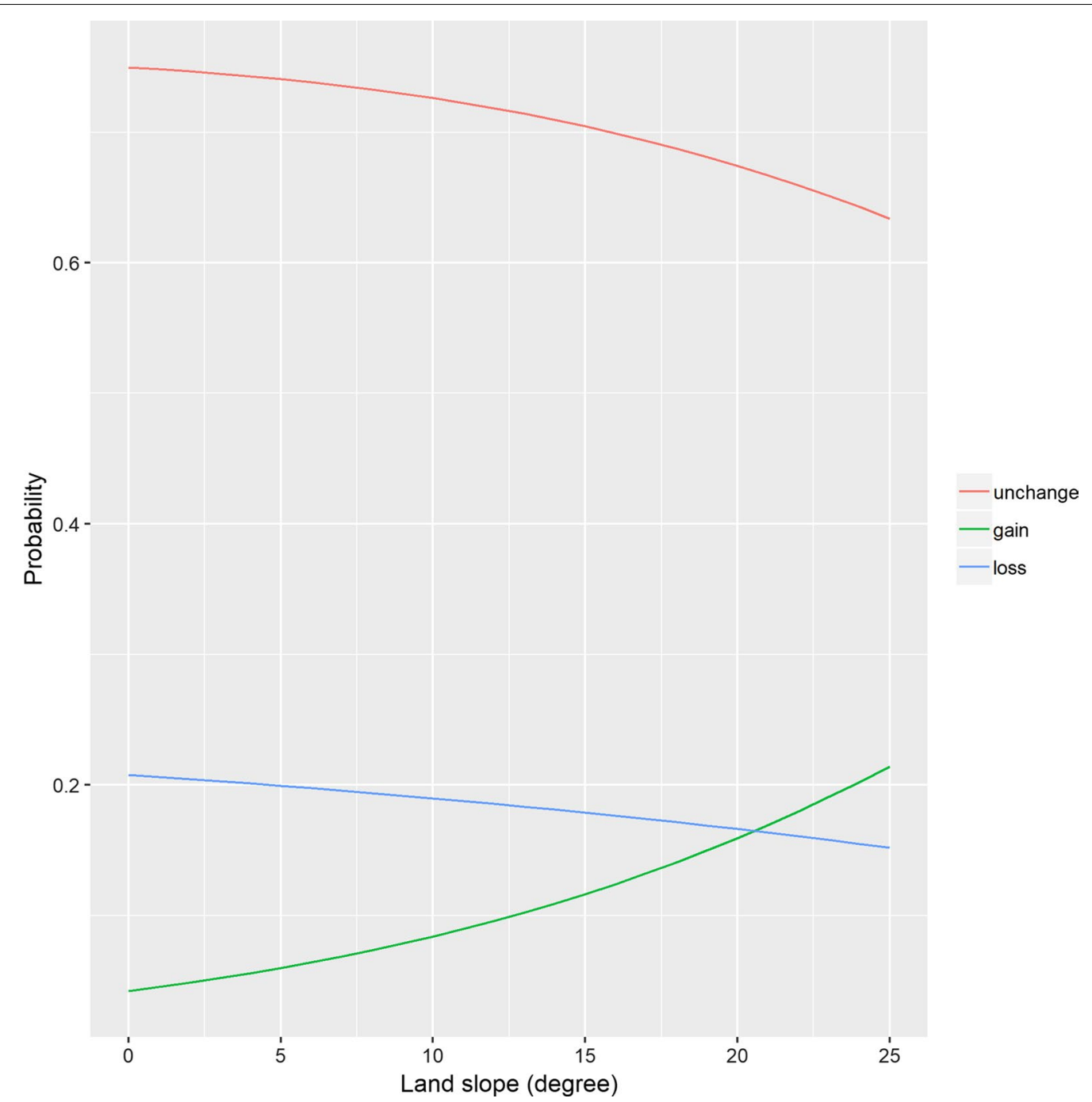

Fig. 2 Predicted probabilities of weight change by land slope in $800 \mathrm{~m}$ network buffer

weight gain or loss for both $400 \mathrm{~m}$ and $800 \mathrm{~m}$ network buffers.

\section{Discussion}

This study sought to identify the effect of environmental attributes specific to rural areas on the risk of longitudinal weight change status among older adults in Japan. This was the first longitudinal study that analyzed the impact of the GIS measured neighborhood environment on the risk of weight change status among older adults in rural areas of Japan. The results of this study showed that hilly neighborhoods were associated with an increased risk of weight gain. The finding was inconsistent with other recent studies which examined the effects of hilly neighborhoods on the risk of diabetes in a cross-sectional fashion [27].
The previous study reported the preventive effect of living in hilly environments for poorly controlled diabetes mellitus among older adults [OR 0.82, (95\% CI 0.70, 0.97)] [27]. The study explained that unconscious training for physical function by walking around the hilly neighborhood was a possible reason for the preventive effect. However, an increase in the degree of steepness may become a barrier for older adults to go out and walk around in their neighborhoods. In fact, the study area of that previous study was not as hilly as our study setting (mean land slope: 3.03 vs. 9.62). Furthermore, one study found a negative association between land slope and the likelihood of engagement in sports among older adults, i.e. living in hilly neighborhood was associated with lower likelihood of sports activity [23]. As we did not analyze to study this possible causal mechanism, further research is necessary, mainly using objectively measured data on 
walking time and physical activity, as well as data on the extent to which older adults go out of the house. The role of this information in mediation must be examined to understand this mechanism.

It was found that almost all other environmental attributes were not significantly associated with weight change status among older adults. While access to public transit was increasingly reported as an important neighborhood environmental attribute for health status in both urban and rural settings [26,33], we did not find an association in our study. This may be because the public transit system should serve people to the extent that they could rely on it for their daily errands, such as commuting, shopping and accessing healthcare. However, public transit does not seem to serve older adults well, based on public transit stop density measures concerning the participants in this study (median, i.e., $400 \mathrm{~m}=1$; median, i.e., $800 \mathrm{~m}=2$ ). Also, the lack of information on whether the participants utilized public transit limited our analysis and, as a result, this study does not reveal the effect of access of public transit on the health of older adults. Residential density (except for the significant negative association of weight gain between the third and first quartiles), intersection density, and the availability of park and recreational centers, which are commonly known as critical environmental attributes for older adults' health in urban settings, were not associated with weight change status in this study. A possible explanation for each attribute is that for residential density, the significant decrease in odds observed between the third and first quartiles is consistent with previous findings [15]. However, as this was only observed within when residential density was categorized, and no dose-response relationship was detected, the finding might have been a result of the sample distribution or arbitrary set neighborhood space. In addition, residential density is a measure of the degree as to how a neighborhood is occupied by residents, thus a key aspect of urban form [34]. Comparing the measures in this study with previous study which focused on an urban setting, it is clear that residential density is considerably low (109.88 vs. 740.3) [35]. In rural settings, it is possible that rather than the simple quantities of housing in neighborhoods, the quality of neighborhoods, manifesting through factors such as social bonding, mutual trust, and prosperity, which are collectively known as social capital, might be more critical for the health of older adults [36]. As rural residents are most likely to move around on their motor vehicles, street connectivity may not be an important factor affecting physical activity and their health, and this finding is consistent with those of previous studies [14]. Parks and recreational center were not associated as they may not be the place for older adults to engage in health-related activities. While this study examined each environmental variable separately, summary index measure is often used in urban neighborhood studies. Walkability index is one of the common indexes which is widely used since it had been developed in 2005 [34]. Although we did not compose such summary index as it was difficult to identify whether each variable have positive or negative effect on weight change based on limited rural studies, it is necessary for future studies to compose index measure that can be generalizable to other rural settings.

One major limitation of this study is that we only analyzed the change in weight, but not in body mass index (BMI) or other more precise body composition measures such as muscle mass. Although our study found an association between land slope and the risk of weight gain, it is controversial whether weight gain among older adults is resulting in an adverse effect on their health. The study conducted a meta-analysis of weight change and all-cause mortality in older adults reported that both weight gain and loss had a higher all-cause mortality risk [pooled Risk Ratio or $R R=1.21$, (95\% CI 1.09, 1.33); pooled RR 1.67, (95\% CI 1.51, 1.85)] [1]. On the other hand, the obesity paradox, which is a phenomenon where BMI-based obesity can be protective or have no effect on specific morbidities or mortality rates from a certain age among older adults has been reported by several population-based cohort studies [37, 38]. Considering the recent evidence of obesity among older adults, future research should use multiple measures, such as weight, BMI, abdominal circumference, and muscle mass, among others.

Another limitation is the relatively small and nonrandomized sample of this study. The participants were limited to those who participated in community health checkups and our Shimane CoHRE study. In addition, we were able to follow up only $32 \%$ of the subjects invited at the baseline. It might have biased our results, and therefore future studies should consider selecting larger samples at random to reduce potential selection bias. Moreover, although we had information on factors such as the attainment of education, data on other socio-economic status (SES) such as income and occupation were not available. The lack of such data might have overestimated the effect of the neighborhood environment or might have failed to detect its effect across SES groups. In developed countries, it is well confirmed that individual SES, including ethnicity, income and education are associated with health outcomes, such as obesity, diabetes and cardiovascular diseases [39, 40]. In order to achieve equity in health, it is necessary to know whether different SES groups could benefit out from policy or environmental interventions, thus, it is critical to include SES as a potential confounder or effect modifier in the studies investigating the effect of neighborhood environments. 
While there is no concern for ethnic disparities as Japanese population is homogenous, lack of information of individual income might have biased our results. Nevertheless, government report that higher educational attainment is associated with higher household income consistently in all age groups in Japan [41]. Furthermore, SES is known to be associated with health related behaviors, i.e., smoking and drinking $[42,43]$, which we could successfully control in our models. We have additionally examined how the effect of neighborhood environment changed before and after including education status in the final models, and found that there was no considerable change of odds of weight gain by slope [ $400 \mathrm{~m}$ buffer without education status: 2 nd vs 1 st quartile: OR 1.266 (95\% CI 0.516, 3.106), 3rd vs 1st: OR 2.234 (95\% CI 1.003, 4.974), 4th vs 1st: OR 2.478 (95\% CI 1.136, 5.405)]. While these could partially compensate for lack of other SES factors, we were unable to assess whether the final sample differed in SES compared with the original sample, which may have hampered the validity of both the models and the final sample. Besides, lack of controlling comorbidities might have biased our results as they might have been confounding for the association neighborhood environment and weight change.

Finally, available data time of environment variables were not exactly the same as the study period, and all environment variables were measured at one time point. In this study, although there are variables that are less likely to change within 3 years, such as land slope and public transit stops, measurement bias for possible longitudinal change of neighborhood environment cannot be neglected. Not capturing all physical environments that can possibly affect weight change, e.g., food environment, also limited our analysis. As weight change occurs by energy balance, i.e., physical activity (energy expenditure) and diet (energy intake), it is important to take into account both aspects in neighborhood studies. A recent systematic review on the association between neighborhood physical environment and adults' weight status pointed out that few studies considered individual diet as a mediator [44]. The major reasons why many studies fail to include diet are that difficulty of obtaining accurate individual diet habit information, and mediation analysis which require complex statistics, such as multilevel structural equation modeling. However, it is critical for future studies need to capture temporal as well as longitudinal data for neighborhood environment, as well as taking into account for both physical activity and diet as mediators to elucidate the mechanism of association between neighborhood environment and weight status. Despite these limitations, this study was significant in that it presented new evidence that the neighborhood environment attributes specific to a rural area, i.e., land slope can affect population health outcomes while focusing on longitudinal weight change status among Japanese older adults. Findings from this study intensify the further needs for both rural and region-specific studies and make a case for the accumulation of more evidence to contribute to the development of evidence-based interventions to facilitate healthy aging.

\section{Conclusion}

In conclusion, a hilly neighborhood was found to be an environmental attribute specific to a rural area with the capacity to result in an increased risk of longitudinal weight gain among older adults in Japan. More regional studies should be carried out in this field with a focus on region-specific environmental attributes that can affect the health of older adults.

\section{Acknowledgements}

We greatly appreciate the cooperation of the participants and we thank CoHRE study member for their skillful assistance. This research was supported by Tobe Maki Foundation (17-JC-002) and the Ministry of Education, Culture, Sports, Science and Technology of Japan (Grants-in-Aid for Scientific Research: 18K11143).

\section{Authors' contributions}

$\mathrm{KO}$ designed the study, prepared and analyzed the data, and was a major contributor in writing this manuscript. TA contributed conception and design of the work, as well as analysis and data interpretation before and throughout the study, and revisions of manuscript. TH contributed the acquisition of longitudinal data, and also contributed the conception of the article in the beginning of the study. MT contributed the acquisition of longitudinal data with TH and also contributed substantially to the interpretation of the data in the preparation. KS and JS contributed to the conception of the study substantially in the beginning of the study, and revisions of manuscript. TN contributed the conception of the study, data acquisition, as well as analysis and interpretation of the analysis results. All authors read and approved the final manuscript.

\section{Funding}

The research was not funded.

\section{Availability of data and materials}

The data used in this paper include personal residential information as well as health related information. Therefore the data are not publicly available due to privacy concerns. The data could be requested and available from the corresponding author on safe manner in accordance with the ethical policy statements of this study protocol.

\section{Ethics approval and consent to participate}

The study protocol and procedure used to secure the informed consent of the participants were approved by the Ethics Committee of Shimane University \#2888.

\section{Consent for publication}

The authors provide full consent for publishing the manuscript.

\section{Competing interests}

The authors declare that they have no competing interests.

\section{Author details}

${ }^{1}$ Center for Community-based Healthcare Research and Education (COHRE), Organization for Research and Academic Information, Shimane University, 223-8 Enya-cho, Izumo-shi, Shimane 693-8501, Japan. ${ }^{2}$ Department of Sports Sociology and Health Sciences, Faculty of Sociology, Kyoto Sangyo University, Motoyama, Kamigamo Kita-ku, Kyoto 603-8555, Japan. ${ }^{3}$ Center for Primary 
Health Care Research, Clinical Research Centre (CRC), Skåne University Hospital, Lund University, Building 28, Floor 11, Jan Waldenströms Gata 35, 205 02 Malmö, Sweden. ${ }^{4}$ Department of Functional Pathology, Faculty of Medicine, Shimane University, 89-1, Enya-cho, Izumo-shi, Shimane 693-8501, Japan.

Received: 22 January 2019 Accepted: 3 May 2019

Published online: 10 May 2019

\section{References}

1. Cheng FW, Gao X, Jensen GL. Weight change and all-cause mortality in older adults: a meta-analysis. J Nutr Gerontol Geriatr. 2015;34(4):343-68.

2. Nanri A, Mizoue T, Takahashi Y, Noda M, Inoue M, Tsugane S. Weight change and all-cause, cancer and cardiovascular disease mortality in Japanese men and women: the Japan Public Health Center-Based Prospective Study. Int J Obes. 2010;34(2):348.

3. Park SY, Wilkens LR, Maskarinec G, Haiman CA, Kolonel LN, Marchand LL. Weight change in older adults and mortality: the Multiethnic Cohort Study. Int J Obes. 2018:42(2):205.

4. Lim SS, Vos T, Flaxman AD, Danaei G, Shibuya K, Adair-Rohani H, et al. A comparative risk assessment of burden of disease and injury attributable to 67 risk factors and risk factor clusters in 21 regions, 1990-2010: a systematic analysis for the Global Burden of Disease Study 2010. The Lancet. 2012;380(9859):2224-60.

5. Wee CC, Phillips RS, Legedza AT, Davis RB, Soukup JR, Colditz GA, Hamel MB. Health care expenditures associated with overweight and obesity among US adults: importance of age and race. Am J Public Health. 2005;95(1):159-65.

6. Qin X, Pan J. The medical cost attributable to obesity and overweight in China: estimation based on longitudinal surveys. Health Econ. 2016;25(10):1291-311

7. Nakamura K, Okamura T, Kanda H, Hayakawa T, Okayama A, Ueshima $\mathrm{H}$, Health Promotion Research Committee of the Shiga National Health Insurance Organizations. Medical costs of obese Japanese: a 10-year follow-up study of National Health Insurance in Shiga, Japan. Eur J Public Health. 2007;17(5):424-9.

8. Yen $\mathrm{H}$, Michael YL, Perdue L. Neighborhood environment in studies of health of older adults: a systematic review. Am J Prev Med. 2009;37(5):455-63.

9. Li F, Harmer P, Cardinal BJ, Bosworth M, Johnson-Shelton D, Moore JM, et al. Built environment and 1-year change in weight and waist circumference in middle-aged and older adults: Portland Neighborhood Environment and Health Study. Am J Epidemiol. 2009;169(4):401-8.

10. Michael YL, Gold R, Perrin N, Hillier TA. Built environment and change in body mass index in older women. Health Place. 2013;22:7-10.

11. Michael YL, Nagel CL, Gold R, Hillier TA. Does change in the neighborhood environment prevent obesity in older women? Soc Sci Med. 2014;102:129-37.

12. Frank LD, Sallis JF, Saelens BE, Leary L, Cain K, Conway TL, Hess PM. The development of a walkability index: application to the Neighborhood Quality of Life Study. Br J Sports Med. 2010;44(13):924-33.

13. Berry N, Coffee N, Nolan R, Dollman J, Sugiyama T. Neighbourhood environmental attributes associated with walking in south Australian adults: differences between urban and rural areas. Int J Environ Res Public Health. 2017;14(9):965.

14. Koohsari MJ, Sugiyama T, Shibata A, Ishii K, Liao Y, Hanibuchi T, et al. Associations of street layout with walking and sedentary behaviors in an urban and a rural area of Japan. Health Place. 2017:45:64-9.

15. Frost SS, Goins RT, Hunter RH, Hooker SP, Bryant LL, Kruger J, Pluto D. Effects of the built environment on physical activity of adults living in rural settings. Am J Health Promot. 2010;24(4):267-83.

16. Eberhardt MS, Pamuk ER. The importance of place of residence: examining health in rural and nonrural areas. Am J Public Health. 2004;94(10):1682-6

17. Martin SL, Kirkner GJ, Mayo K, Matthews CE, Durstine JL, Hebert JR. Urban, rural, and regional variations in physical activity. J Rural Health. 2005;21(3):239-44.

18. Christiansen LB, Cerin E, Badland H, Kerr J, Davey R, Troelsen J, et al. International comparisons of the associations between objective measures of the built environment and transport-related walking and cycling: IPEN adult study. J Transp Health. 2016;3(4):467-78.

19. Oba T, Matsunaka R, Nakagawa D, Inoue K. Analysis of the relationship between urban characters and physical activity levels based on the travel behavior data. J City Plan Inst Jpn 2013;48(1):73-81.

20. Sallis JF, Cerin E, Conway TL, Adams MA, Frank LD, Pratt M, et al. Physical activity in relation to urban environments in 14 cities worldwide: a crosssectional study. The Lancet. 2016;387(10034):2207-17.

21. Baernholdt M, Yan G, Hinton I, Rose K, Mattos M. Quality of life in rural and urban adults 65 years and older: findings from the National Health and Nutrition Examination Survey. J Rural Health. 2012;28(4):339-47.

22. Statistics Bureau. Portal site of official statistics of Japan. https://www.estat.go.jp/en/stat-search/files?page $=1 \&$ query $=2015 \&$ layout $=$ datas et\&toukei $=00200524 \&$ tstat $=000000090001 \&$ cycle $=7 \&$ month $=0 \&$ tclas s1 $=000001011679$ (2015). Accessed 1 Oct 2018.

23. Hanibuchi T, Kawachi I, Nakaya T, Hirai H, Kondo K. Neighborhood built environment and physical activity of Japanese older adults: results from the Aichi Gerontological Evaluation Study (AGES). BMC Public Health. 2011;11(1):657.

24. Hanibuchi T, Kondo K, Nakaya T, Nakade M, Ojima T, Hirai H, Kawachi I. Neighborhood food environment and body mass index among Japanese older adults: results from the Aichi Gerontological Evaluation Study (AGES). Int J Health Geogr. 2011;10(1):43.

25. Hamano T, Shiotani Y, Takeda M, Abe T, Sundquist K, Nabika T. Is the effect of Body Mass Index on hypertension modified by the elevation? A crosssectional study of rural areas in Japan. Int J Environ Res Public Health. 2017;14(9):1022.

26. Kamada M, Kitayuguchi J, Inoue S, Kamioka H, Mutoh Y, Shiwaku K. Environmental correlates of physical activity in driving and non-driving rural Japanese women. Prev Med. 2009;49(6):490-6.

27. Fujiwara T, Takamoto I, Amemiya A, Hanazato M, Suzuki N, Nagamine $Y$, et al. Is a hilly neighborhood environment associated with diabetes mellitus among older people? Results from the JAGES 2010 study. Soc Sci Med. 2017;182:45-51.

28. Koohsari M, Nakaya T, Oka K. Activity-friendly built environments in a super-aged society, Japan: current Challenges and toward a Research Agenda. Int J Environ Res Public Health. 2018;15(9):2054.

29. Ministry of Land Infrastructure Transport and Tourism. Land and Climate of Japan. http://www.mlit.go.jp/river/basic_info/english/land.html (2007). Accessed 1 Oct 2018.

30. Statistics Bureau Home Page/What is a Densely Inhabited District? https ://www.stat.go.jp/english/data/chiri/did/1-1.html (1996). Accessed 15 Mar 2019.

31. Statistics Bureau. Portal site of official statistics of Japan. https://www.estat.go.jp/en/stat-search/files?page $=1 \&$ layout $=$ datalist $\&$ touke $=00200$ $521 \&$ tstat $=000001080615 \&$ cycle $=0 \&$ tclass $1=000001089055 \&$ tclas s2 $=000001089057 \&$ tclass3 $=000001089091 \&$ survey $=$ population\&resul t_page $=1 \&$ second $=1 \&$ second $2=1$ (2015). Accessed 1 Oct 2018.

32. de Mutsert R, Sun Q, Willett WC, Hu FB, van Dam RM. Overweight in early adulthood, adult weight change, and risk of type 2 diabetes, cardiovascular diseases, and certain cancers in men: a cohort study. Am J Epidemiol. 2014;179(11):1353-65.

33. Todd M, Adams MA, Kurka J, Conway TL, Cain KL, Buman MP, et al. GISmeasured walkability, transit, and recreation environments in relation to older Adults' physical activity: a latent profile analysis. Prev Med. 2016;93:57-63

34. Frank LD, Schmid TL, Sallis JF, Chapman J, Saelens BE. Linking objectively measured physical activity with objectively measured urban form: findings from SMARTRAQ. Am J Prev Med. 2005;28(2):117-25.

35. Hinckson E, Cerin E, Mavoa S, Smith M, Badland H, Stewart T, et al. Associations of the perceived and objective neighborhood environment with physical activity and sedentary time in New Zealand adolescents. Int J Behav Nutr Phys Act. 2017;14(1):145.

36. Wu YH, Moore S, Dube L. Social capital and obesity among adults: Iongitudinal findings from the Montreal neighborhood networks and healthy aging panel. Prev Med. 2018;111:366-70.

37. Tamakoshi A, Yatsuya H, Lin Y, Tamakoshi K, Kondo T, Suzuki S, et al. BMl and all-cause mortality among Japanese older adults: findings from the Japan collaborative cohort study. Obesity. 2010;18(2):362-9.

38. Yamazaki K, Suzuki E, Yorifuji T, Tsuda T, Ohta T, Ishikawa-Takata K, Doi $\mathrm{H}$. Is there an obesity paradox in the Japanese elderly population? A 
community-based cohort study of 13280 men and women. Geriatr Gerontol Int. 2017;17(9):1257-64.

39. Agborsangaya CB, Lau D, Lahtinen M, Cooke T, Johnson JA. Multimorbidity prevalence and patterns across socioeconomic determinants: a cross-sectional survey. BMC Public Health. 2012;12(1):201.

40. Katikireddi SV, Skivington K, Leyland AH, Hunt K, Mercer SW. The contribution of risk factors to socioeconomic inequalities in multimorbidity across the lifecourse: a longitudinal analysis of the Twenty-07 cohort. BMC Med. 2017;15(1):152.

41. Ministry of Health, Labour and Welfare. Basic Survey on Wage Structure, page 6, Land and Climate of Japan. https://www.mhlw.go.jp/english/ database/db-l/wage-structure.html (2012). Accessed 20 Mar 2019.

42. Lantz PM, House JS, Lepkowski JM, Williams DR, Mero RP, Chen J. Socioeconomic factors, health behaviors, and mortality: results from a nationally representative prospective study of US adults. JAMA. 1998;279(21):1703-8

43. Hanibuchi T, Nakaya T, Honjo K. Trends in socioeconomic inequalities in self-rated health, smoking, and physical activity of Japanese adults from 2000 to 2010. SSM Popul Health. 2016;2:662-73.

44. Mackenbach JD, Rutter H, Compernolle S, Glonti K, Oppert JM, Charreire $\mathrm{H}$, et al. Obesogenic environments: a systematic review of the association between the physical environment and adult weight status, the SPOTLIGHT project. BMC Public Health. 2014;14(1):233.

\section{Publisher's Note}

Springer Nature remains neutral with regard to jurisdictional claims in published maps and institutional affiliations.
Ready to submit your research? Choose BMC and benefit from:

- fast, convenient online submission

- thorough peer review by experienced researchers in your field

- rapid publication on acceptance

- support for research data, including large and complex data types

- gold Open Access which fosters wider collaboration and increased citations

- maximum visibility for your research: over $100 \mathrm{M}$ website views per year

At BMC, research is always in progress.

Learn more biomedcentral.com/submissions 